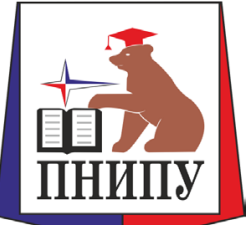

PNRPU MECHANICS BULLETIN

№ 3-4, 2018

http://vestnik.pstu.ru/mechanics/about/inf/

UDC 539.3

\title{
IDENTIFICATION METHOD OF GRADIENT MODELS PARAMETERS OF INHOMOGENEOUS STRUCTURES BASED ON DISCRETE ATOMISTIC SIMULATIONS
}

\author{
S.A. Lurie ${ }^{1,2}$, Y.O. Solyaev ${ }^{1,2}$ \\ ${ }^{1}$ Institute of Applied Mechanics RAS, Moscow, Russian Federation \\ ${ }^{2}$ Computing Centre RAS, Moscow, Russian Federation
}

\section{ARTICLE INFO}

Received: 18.08.2014

Accepted: 10.09 .2014

Published: 25.12.2018

\section{Keywords:}

gradient theory, gradient moduli, criterion of symmetry,

one-parametrical model, layered composite, discrete atomistic modelling, scale parameter, identification.

\begin{abstract}
In this paper, the characteristics, features and the corresponding boundary value problems of gradient theories of elasticity are examined. A brief description on one-parametric applied model, which is one of the several variants of the gradient elasticity theories, is given. In relation to that, we represent a continuum gradient model of two-phase composite structures that will allow us to evaluate the influence of scale parameters on their effective mechanical properties.

In identifying the additional physical parameters of gradient elasticity models, a new method is introduced where a comparison between the results of continuum and discrete-atomistic modeling for specific tested heterogeneous structures is made. As a result, a procedure and the respective algorithm defining the additional parameter of applied gradient continuum model of heterogeneous media is proposed; and in such procedure, an interphase zone is characterized at the contact surface of a two-phase composite and the scale effects represented by cohesions-interaction fields, which are localized near to the boundaries of contact surfaces, are defined. This additional physical parameter of gradient model is found through parameters of potentials, which are used to describe the specific studied structure in the discrete-atomistic modeling.

To justify and validate the proposed method, a numerical investigation is conducted and comparison is made between the results of continuum and discrete-atomistic modeling. The examination reveals that a high degree of accuracy of prediction can be provided by the continuum one-parametric gradient theory when describing the effective properties of countable multiple set of two-phase heterogeneous studied structures, which are formed by atomic substructures with various properties (various parameters of potentials).

Finally, it is demonstrated that the identification method of parameters in gradient elasticity theories for heterogeneous structures is well described by Leonard-John potential and Morse potential. Furthermore, we consider that when the parameters of potentials are known, the various types of cross interactions of atoms can be treated as 'ideal' or 'damaged' interactions as per Lorentz-Berthelot's rule.
\end{abstract}




\section{Introduction}

Historically, development of the gradient theories of elasticity began thanks to the path breaking works [1-4]. In contrast to the classical theory of elasticity which does not take into account any scale parameters in constitutive equations, the gradient theories of deformation include parameters of length dimension and, therefore, are suitable for simulation of scale effects.

Currently, the gradient theories of elasticity are widely used to describe dimensional effects and non-local behavior observed in semi-crystalline and nanostructured materials, geomaterials, and biomaterials as well as to simulate anomalous properties of hyperfine structures (micro and nanoparticles, hyperfine cantilever beams and plates, carbon nanotubes and metal nanowires) [5-9]. Dimensional effects in hyperfine structures were observed experimentally, for example, in bend tests of aluminum rods, epoxy and polypropylene cantilever beams [10-12]. The gradient theories of elasticity are used for evaluation of the effective properties of filled composites with micro/nanoinclusions to take into account so-called secondary effects [13-20]. The theories are also used to evaluate conformance between the discrete atomic simulation level and the continual simulation level used in continuum mechanics [21].

Generally, simulation of scale effects in various physicomechanical processes is performed using the secondary gradient theories due to their relative simplicity $[8,18,22,23]$. In the secondary gradient theory, the Cauchy stress tensor $\sigma$ and the couple stress pseudotensor $\mu$ are arcwise connected to the strain tensor $\varepsilon$ and the strain gradient tensor $\chi$. In general, there are 300 independent material coefficients based on the symmetry conditions (the gradient theory of deformations with a symmetric strain tensor), while the number of physical constants decreases to 7 for isotropic centrosymmetric materials (see, for example, $[2,5,6])$. On the one hand, the large number of material coefficients underlines richness of the gradient theories, but on the other hand, it points to the obvious difficulties in determining physical parameters from the available set of experimental studies. It should be noted that in order to use the gradient theory for simulation in specific theoretical and engineering problems, physical constants must be determined fairly accurately and reliably. Consequently, even a correctly developed theory, determined up to a large number of physical constants that are impossible to derive from experimental trials accurately, becomes untenable in relation to specific applications. Therefore, the problem of additional physical parameters determination in the gradient theories of elasticity is very relevant. It should be noted that in some cases this problem can be solved using indirect methods, for example, based on comparison of theoretical results with experimental data on the effective properties of the nanocomposites filled with rigid nanoparticles [18].

In this paper, we discuss direct evaluation of the additional physical constant responsible for gradient (scale) effects in applied versions of the gradient theory of elasticity. We develop a method to determine the nonclassic physical constant based on comparison of the results of direct discrete atomic simulation of heterogeneous structures and the results of continual simulation carried out using the one-parameter gradient theory. The direct feasibility demonstration of the proposed methodology is the results of preliminary studies [21] showing that the gradient theory gives a very accurate description of the onedimensional composite chains properties. Simulation of the effective properties of that chains was carried out on the basis of direct discrete atomic simulation using the LennardJones potential (6-12).

The article develops the methodology based on consideration of the one-dimensional discrete model of composite system and the one-dimensional continual composite model respectively. In the case under consideration, the continual solution, taking into account scale effects, obtained for periodic composite structures using the gradient theory of elasticity, is presented in an analytical form which helps to determine the nonclassic physical constant. Therein, the discrete model may be created using, formally, any potential that is most suitable for simulation of the materials under consideration.

\section{Gradient Theories of Elasticity}

Let us give a brief introduction to the gradient theories of elasticity and introduce variants of the applied oneparameter theories of elasticity. We use the variational formulation to define the linear gradient theory of elasticity in displacements for isotropic centrosymmetric materials:

$$
\begin{gathered}
E=\frac{1}{2} \int C_{i j k l} R_{i, j} R_{k, l} d V+\frac{1}{2} \int C_{i j k l m n} R_{i, j k} R_{l, m n} d V- \\
-\int f_{i} R_{i} d V-\oint\left(t_{i} R_{i}+q_{i} R_{i, j} n_{j}\right) d S .
\end{gathered}
$$

Where $R_{i}$ is the displacement vector; $f_{i}, t_{i}$, and $q_{i}$ are vectors of given forces in the volume of a body and on its surface; $n_{i}$ is the normal to the body surface at the point under consideration; $C_{i j k l}$ and $C_{i j k l m n}$ are the moduli tensor of the classic elasticity theory and the hexor of gradient moduli for isotropic materials accordingly.

Tensor components of ordinary and couple stresses are determined by the Green equalities:

$$
\begin{gathered}
\sigma_{i j}=\partial E / \partial R_{i, j}=C_{i j k l} R_{k, l}, \\
\mu_{i j k}=\partial E / \partial R_{i, j k}=C_{i j k l m n} R_{l, m n}
\end{gathered}
$$

Couple stresses tensor taking into account the "moment" interaction in the body volume.

It is assumed that tensors of classic and gradient moduli meet the following necessary potential conditions:

$$
C_{i j k l}=C_{k l i j}, \quad C_{i j k l m n}=C_{l m n i j k} .
$$

For the gradient theory of deformation that is more particular than the gradient theory of distortion, it is necessary to introduce the following additional symmetry conditions for the first pair of indices: 


$$
C_{i j k l m n}=C_{j i k l m n} .
$$

For the isotropic theory of elasticity, the tensor of elasticity moduli $C_{i j k l}$ is determined by the Lame coefficients $\lambda, \mu: C_{i j k l}=\lambda \delta_{i j} \delta_{k l}+\mu\left(\delta_{i k} \delta_{j l}+\delta_{i l} \delta_{j k}\right)$ where $\delta_{i j}$ is the bivalent mixed tensor. By varying the potential energy functional (1), we obtain the equilibrium equations and natural static boundary conditions for the gradient theory of elasticity:

$$
\begin{gathered}
\sigma_{i j, j}-\mu_{i j k, k j}+f_{i}=0, \\
\mu_{i j k} n_{j} n_{k}=q_{i}, \\
\sigma_{i j} n_{j}-\mu_{i j k, k} n_{j}-\left(\mu_{i j k} n_{k}\right)_{, j}+\left(\mu_{i j k} n_{j} n_{k}\right)_{, l} n_{l}=t_{i} .
\end{gathered}
$$

The system of resolving equations has a higher degree than the Lame equations of the classic elasticity theory. In addition to the forces on the surface, the non-classic boundary conditions for the couple stresses allow to take into account the influence of given external moment factors applied to the boundary of the body surface.

The equations (6) in contact problems are complemented by continuity conditions of the displacements and their first derivatives.

\section{Principle of Symmetry in Gradient Theories of Elasticity}

We take into account that since the distortion tensor $R_{i, j} \quad$ is assumed to be continuous in the variational formulation (1), there is independence from the differentiation order of the displacement vector $R_{i, j k}=R_{i, k j}$, and the tensor of the second derivatives of displacements $\chi_{i j k}=R_{i, j k}$ is symmetric for the last two indices. Consequently, the equality (3) results in the additional symmetry conditions for the tensor of gradient moduli:

$$
C_{i j k l m n}=C_{i k j l m n}, \quad C_{i j k l m n}=C_{i j k l n m} .
$$

It should be noted that the antisymmetric part of the gradient elasticity moduli $\left(C_{i j k l m n}-C_{i k j l m n}\right) / 2$ may be arbitrary in the defining relation for the couple stresses $\mu_{i j k}$ in (3). It is also easy to see that the antisymmetric part of the gradient moduli is also not included in the expression for the potential energy density $\left(C_{i j k l m n} R_{i, j k} R_{l, m n}\right) / 2$. Consequently, it can be said that the asymmetric components of the gradient tensors $\left(C_{i j k l m n}-C_{i k j l m n}\right) / 2$ are energetically invisible. It can be shown that the asymmetric part of the gradient elasticity tensors can also be ignored when we formulate the resolving equilibrium equations (5) for displacements. However, it is important to note that the asymmetric part of the gradient elasticity tensors can make a parasitic input to the static boundary conditions (6): $\sigma_{i j} n_{j}-\mu_{i j k, k} n_{j}-\left(\mu_{i j k} n_{k}\right)_{, j}+\left(\mu_{i j k} n_{j} n_{k}\right)_{, l} n_{l}=t_{i}$ and should be excluded. Therefore, the symmetry conditions for the gradient moduli tensor (7) in the gradient theory of elasticity are the criteria of accuracy and should be included in the additional necessary conditions for the elasticity tensors. The symmetry criterion of the gradient theories was defined for the first time with the help of Prof. A. Gusev (ETH, Zurich).

\section{Applied Gradient Theories of Elasticity}

Papers [2-4] show that the strain energy density $\bar{E}$ of the gradient theory $E=\int \bar{E} d V$ for an isotropic body can be formulated using five additional gradient elasticity moduli $c_{1}, c_{2}, c_{3}, c_{4}, c_{5}$ in the following way:

$$
\begin{gathered}
\bar{E}=\frac{1}{2}\left[C_{i j k l} \varepsilon_{i j} \varepsilon_{k l}+C_{i j k l m n} R_{i, j k} R_{l, m n}\right]= \\
=\frac{1}{2} C_{i j k l} \varepsilon_{i j} \varepsilon_{k l}+c_{1} \theta_{, i} \nabla^{2} R_{i}+c_{2} \theta_{, i} \theta_{, i}+ \\
+c_{3} \nabla^{2} R_{i} \nabla^{2} R_{i}+c_{4} R_{i, j k} R_{i, j k}+c_{5} R_{i, j k} R_{j, i k} .
\end{gathered}
$$

It is easy to define that the elasticity moduli $C_{i j k l m n}$ for the gradient theory of deformations (the first two indices (4) are symmetrical) can also be expressed by five independent physical constants $c_{1}, c_{2}, c_{3}, c_{4}, c_{5}$ in the representation (8). However, in general, the tensor of the gradient elasticity moduli does not meet the symmetry criterion (7). Using additional conditions of symmetry (7), it can be established that, in the case of complete symmetry in the Mindlin model, there are the following relations: $c_{1}=4 c_{3}, c_{1}=c_{2}$, $c_{5}=2 c_{4}$. Therefore, only two physical constants are independent additional parameters: $c_{3}$ and $c_{4}$. In this case, the expression for the couple stresses $\mu_{i j k}$ takes the following form:

$$
\begin{gathered}
\mu_{i j k}=c_{3}\left[\nabla^{2}\left(\delta_{i j} R_{k}+\delta_{i k} R_{j}+\delta_{j k} R_{i}\right)+\right. \\
\left.+2\left(\delta_{i j} \theta_{, k}+\delta_{i k} \theta_{, j}+\delta_{j k} \theta_{, i}\right)\right]+c_{4}\left(R_{k, i j}+R_{j, i k}+R_{i, j k}\right) .
\end{gathered}
$$

As the result, the symmetry conditions (7) make it possible to reduce the number of independent additional constants to two. In this case, the variational formulation gives the following resolving equations in displacements:

$$
\begin{gathered}
H_{i j} L_{j k} R_{k}+f_{i}=0, H_{i j}=\delta_{i j}-l_{1}^{2} \delta_{i j} \nabla^{2}-l_{2}^{2} \partial_{i} \partial_{j}, \\
L_{j k}=\mu \delta_{j k} \nabla^{2}+(\lambda+\mu) \partial_{j} \partial_{k}, \\
l_{1}^{2}=\left(c_{3}+c_{4}\right) / \mu, \\
l_{2}^{2}=\left[\mu\left(c_{1}+c_{2}+c_{5}\right)-(\lambda+\mu)\left(c_{3}+c_{4}\right)\right] /[\mu(\lambda+2 \mu)] .
\end{gathered}
$$

These equations are expressed using two scale parameters $l_{1}^{2}, l_{2}^{2}$.

The one-parameter gradient theory of deformation, proposed in the papers [8, 22, 23], is widely used for 
applied problems. In this kind of theory, the density of the gradient energy of deformation has the following form:

$$
\bar{E}=(1 / 2) C_{i j k l} \varepsilon_{i j} \varepsilon_{k l}+l^{2}\left[(1 / 2) \lambda \theta_{, k} \theta_{, k}+\mu \varepsilon_{i j, k} \varepsilon_{i j, k}\right] \text {. }
$$

This theory can be formally obtained from the model (1)-(6) assuming that

$$
c_{1}=c_{3}=0, c_{2}=(1 / 2) l^{2} \lambda, c_{4}=c_{5}=(1 / 2) l^{2} \mu .
$$

The system of resolving equations for the applied model [22, 23]:

$$
\left(1-l^{2} \Delta\right) L_{i j} R_{j}+f_{i}=0
$$

It should be noted that this theory never meets the symmetry condition (7), i.e. it is not adequate enough.

Another example of a one-parameter model is the gradient model of the interface layer [18-20] where the density of the gradient energy of deformation is defined through the additional scale parameter $C$ (cohesive modulus) using the following formula:

$$
\bar{E}=(1 / 2)\left[C_{i j k l} \varepsilon_{i j} \varepsilon_{k l}+\left(L_{j k} R_{k}\right)\left(L_{j k} R_{k}\right) / C\right] .
$$

This theory corresponds to the following relation between physical constants in the Mindlin model $c_{1}, c_{2}, c_{3}, c_{4}, c_{5}$ and the scale parameters $l_{1}$ and $l_{2}$ :

$$
\begin{aligned}
& c_{1}=2 \mu(\mu+\lambda) / C, \quad c_{2}=(\mu+\lambda)^{2} / C, \\
& c_{3}=\mu^{2} / C, \quad c_{4}=c_{5}=0, \quad l_{1}^{2}=(1-2 v) l_{2}^{2},
\end{aligned}
$$

where $v$ is the Poisson ratio.

The system of resolving equations (equilibrium equations) for the gradient theory of the interface layer has the following form:

$$
-\frac{1}{C}\left(L_{i j}-C \delta_{i j}\right) L_{j k} R_{k}+f_{i}=0 .
$$

It should be noted here that the model of the interface layer (14) meets the symmetry criterion being a gradient theory of distortion, but not a theory of deformation.

Hereinafter, we will use the solution of the onedimensional problem of the gradient elasticity theory to implement the procedure of additional parameter identification in the gradient theory of elasticity. In fact, we need to formulate a gradient model of a stratified medium to do this. It is easy to make sure that all the applied gradient theories (8)-(10), (11)-(13) discussed above and the interface layer theory (14), (15) give equivalent formulations for the one-dimensional problem. The resolving equation for the one-dimensional problem has the following form:

$$
\begin{gathered}
L L_{C}(R)=0, \quad L(R)=(2 \mu+\lambda)\left(\partial^{2} R / \partial x^{2}\right), \\
L_{C}(R)=(2 \mu+\lambda)\left(\partial^{2} R / \partial x^{2}\right)-C R .
\end{gathered}
$$

The boundary conditions result from the variational formulation. For kinematic boundary conditions, they are formulated relatively to the displacements $R$ derived from the displacements $\partial R / \partial x$, and in the case of static boundary conditions, the boundary conditions are formulated relatively to the couple stresses $[(2 \mu+\lambda) / C] L(R)$ and stresses $[(2 \mu+\lambda) / C] \partial L_{C}(R) / \partial x$.

Now let us consider the one-dimensional model of the stratified medium. In this case, the boundary contact conditions for a representative periodicity fragment are formulated with the periodicity jump condition and can be represented in the following form:

$$
\begin{gathered}
U\left(-d_{1}-d_{2}\right)=U\left(d_{1}+d_{2}\right)-2\left(d_{1}+d_{2}\right), \\
\frac{\partial U\left(-d_{1}-d_{2}\right)}{\partial x}=\frac{\partial U\left(d_{1}+d_{2}\right)}{\partial x}, \\
u\left(-d_{1}-d_{2}\right)=u\left(d_{1}+d_{2}\right), \\
\frac{\partial u\left(-d_{1}-d_{2}\right)}{\partial x}=\frac{\partial u\left(d_{1}+d_{2}\right)}{\partial x} .
\end{gathered}
$$

Where $U$ and $u$ are two components of the general displacement field $R=U-u$ which meet the conditions of the classic equation $L(U)=0$ and cohesive field equation $L_{C}(u)=0$ respectively: $U=-L_{C}(R) / C \quad u=-L(R) / C \quad 2 d_{2}$ is the length of the inclusion, $d_{1}$ is the length of the matrix layer to the right and left of the inclusion (Fig. 1), the total length of the matrix layer in the representative fragment of the stratified system is $2 d_{1}$.

Solution of the boundary problem (16)-(18) can be obtained in a clear analytical form and expressed by three functions in different increment ranges of the argument: $R=R_{1}(x), \quad-d_{1}-d_{2}<x<-d_{2}, \quad R=R_{2}(x), \quad-d_{2}<x<d_{2}$, $R=R_{3}(x), \quad d_{2}<x<d_{1}+d_{2}$, where

$$
\begin{gathered}
R_{1}(x)=H_{1} x+\left(H_{1}-1\right)\left(d_{1}+d_{2}\right)-d_{1} \operatorname{sh} \kappa_{1}\left(x+d_{1}+d_{2}\right), \\
R_{2}(x)=H_{2} x-d_{2} \operatorname{sh~} \kappa_{2} x, \\
R_{3}(x)=H_{1} x-\left(H_{1}-1\right)\left(d_{1}+d_{2}\right)-d_{1} \operatorname{sh} \kappa_{1}\left(x-d_{1}-d_{2}\right),
\end{gathered}
$$

Where $\quad \kappa_{1,2}=1 / l_{1,2}=\sqrt{C_{1,2} / k_{1,2}}, \quad k_{1,2}=2 \mu_{1,2}+\lambda_{1,2} ;$ index 1 refers to the matrix phase, index 2 refers to the inclusion phase.

The effective elasticity modulus $\hat{E}$ for the stratified medium is determined according to the asymptotic averaging method on the basis of the solution (19) as average stresses of the periodicity cell of the classic component of the solution $U(x)$. As for the model in question, this gives the following expression for $\hat{E}$ :

$$
\hat{E}=E_{1} E_{2}\left(d_{1}+d_{2}\right) /\left[E_{1} d_{2}+E_{2} d_{1}-\frac{\left(E_{1}-E_{2}\right)^{2}}{\left(E_{1} \alpha_{2}+E_{2} \alpha_{1}\right)}\right] \text {. }
$$

where $\alpha_{1,2}=\kappa_{1,2} / \operatorname{th}\left(\kappa_{1,2} d_{1,2}\right)$. 
The expression (20) comes to the classical Reiss formula for the lack of any gradient effects $\left(l_{1}=0, l_{2}=0\right)$.

\section{Methodology for Identification of Gradient Model Scale Parameters Based on Molecular Dynamics Simulation}

We consider two one-dimensional models of composite discrete and continual. The one-dimensional composite (layered composite) is a periodic structure described by a representative fragment - the periodicity cell shown in Fig. 1. Then, we compare the effective elasticity modulus, calculated for the discrete composite structure and consisting of $2 n_{1}$ atoms of the first phase and $n_{2}$ atoms of the second phase, with the results of calculation of the layered composite effective elasticity modulus using the continual gradient model (see Fig. 1). The lengths of the continual cells of each phase correspond to the lengths of the discrete atom chains simulating these phases. The phase length for the discrete composite model is calculated using equilibrium interatomic distances. In turn, the equilibrium distances for the discrete model are defined by minimization the potential energy calculated using given interaction potentials. As the result, the discrete fragment corresponds to the one-dimensional continual fragment of the composite.

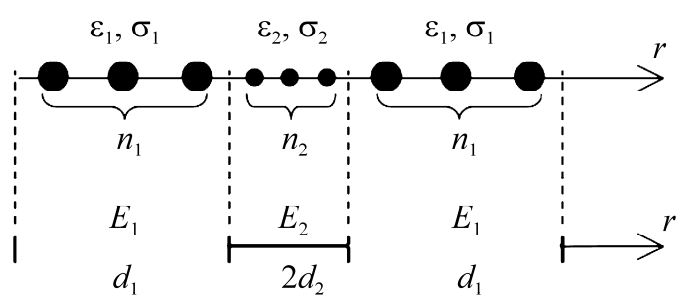

Fig. 1. Discrete and continual models of the two-component composite structure

We use the Lennard-Jones (6-12) and Morse potentials to simulate interatomic interactions:

$$
\begin{gathered}
U(r)=4 \varepsilon\left(\left(\frac{\sigma}{r}\right)^{12}-\left(\frac{\sigma}{r}\right)^{6}\right), \\
U(r)=\varepsilon\left(\mathrm{e}^{-2 \alpha(r-\sigma)}-2 \mathrm{e}^{-\alpha(r-\sigma)}\right),
\end{gathered}
$$

Parameters of interaction between atoms of different types will be determined according to the Lorentz-Berthelot rule [24]:

$$
\varepsilon_{12}=\chi \sqrt{\varepsilon_{1} \varepsilon_{2}}, \sigma_{12}=\frac{\sigma_{1}+\sigma_{2}}{2}, \alpha_{12}=\sqrt{\alpha_{1} \alpha_{2}},
$$

where $\chi$ varies from 0 to 1 , and the parameters $\varepsilon_{1}, \sigma_{1}$ and $\varepsilon_{2}, \sigma_{2}$ determine potential characteristics of two atom types.

Equilibrium arrangement of atoms is found by determining the minimum value of the potential energy of the considered atomic system. It should be noted that in the case of the "nearest neighbors" model, which assumes interaction only between two neighboring atoms, the equilibrium interatomic distances are determined analytically using known parameters of the interatomic interaction potentials. The elasticity modulus is defined using the relation connecting the increment of the potential energy with the effective modulus: $\Delta U=\hat{E}\left(d_{1}+d_{2}\right) / 2$, where $\Delta U$ is the increment of the potential energy of the fragment when the periodicity cell is under unit strain.

In the case of a composite chain, the procedure does not change, and the obtained value is the effective elasticity modulus of the one-dimensional composite taking into account not only the volume content of the phases $\left(n_{1} / n_{2}\right.$ ratio), but also the dimensional factors ( $n_{1}$ or $n_{2}$ values) which are natural dimensional parameters of the discrete structure. They describe the number of interface boundaries and the length of the interface layer.

It should be noted that the scale effects are substantial for the materials where properties of the phases (in this case, the elasticity moduli) are significantly different. Polymer composites reinforced with carbon nanoinclusions (nanotubes), where moduli of elasticity can differ by more than 80 times, may serve as an example. For the structures we considered in this work, the elasticity moduli of the phases differ by no more than ten times, therefore, the scale effects here are not so pronounced. Taking into account these effects that can be directly simulated in the frameworks of the one-dimensional discrete model, we can accurately predict anomalous change of the properties of micro/nanostructures for the composites under consideration.

The identification method for parameters of the gradient models is based on comparison of the calculation results of the effective elasticity moduli defined using discrete and continual simulation. Comparison of the solutions is performed according to the following algorithm:

1. Choose the type of interatomic interaction potential. It should be noted that, in general, the methodology allows to work with any potential. Potentials for the simulation are chosen based on preliminary physical and chemical studies and should be consistent with the structures and materials under consideration. Taking into account these requirements, we use the Lenard-Jones (6-12) and Morse potentials in this work.

2. Specify the number of atoms in the chain and their type, i.e. choose values of the potential parameters. In this work, values of the potential parameters were taken from [24-26].

3. Determine equilibrium distances and values of the elasticity moduli for each of the homogeneous atom chains that form the composite chain. These values are used as initial data - the moduli of elasticity and phase lengths in the formula (20). The calculated moduli of the chains correspond to the idealized one-dimensional atomic structures under consideration. Generally speaking, they differ from the real properties of the three-dimensional structures of the materials. Nevertheless, the algorithm for 
determination of the scale parameters based on corresponding monoatomic structures can be used for identification of gradient models and evaluation of properties of the composite materials under consideration.

4. Determine the effective rigidity of the chain in the frameworks of the discrete model .

5. Determine the scale parameter of the continual model so the effective modulus, obtained in the continual model for the chain using the formula (20), is equal to the modulus obtained in the frameworks of the discrete model for all parameter values of the structure $n_{1}$ (or $n_{2}$ ) and $n_{1} / n_{2}$. Whereby, we assume that the structure under consideration (i.e. both phases) is described by the single scale parameter $l_{1}=l_{2}=l$ in continual simulation.

In the general case, there is one characteristic that takes into account typical size of the structure for each phase in the two-component composite. However, we believe that these structure characteristics of the different phases are identical. In other words, we assume that the twocomponent composite has single scale parameter $l_{1}=l_{2}=l$ in the relations (19), (20) in continual simulation. Further direct calculations, carried out using discrete simulation, showed that, with this simplification, the proposed procedure for identification of the single scale parameter of the two-component composite makes it possible to describe the entire set of composites for specific phases with extremely high accuracy. The physical meaning of the result is that the obtained single scale parameter $(l)$ adequately describes the length of the interface layer located in the vicinity of the phase contact boundary of the composite structure.

6. Vary the parameters $n_{1}$ and $n_{2}$ to make sure that the value of the scale parameter $l$ allows to obtain coincidence of the solutions of the continual and discrete models for any chains created from atoms of the selected type.

It is important to note that the main feature of the proposed gradient parameter identification method lies in the fact that it is always possible to find the scale parameter that allows to describe the entire countable set of onedimensional composites with high accuracy.

\section{Calculation Results and Examples of Gradient Model Parameters Identification}

Let us consider examples of solution for the identification problem of the non-classic parameter, determining the typical size of a particular structure, using examples of two types of composites formed from phases of silicon and carbon atoms and lead and iron atoms respectively. In this case, both phases of the composite $(S i)_{n_{1}}-(C)_{n_{2}}$ are well described by the Lenard-Jones potential $[24,25]$, and interactions of atoms in the phases of the second composite, consisting of iron and lead atoms $(\mathrm{Fe})_{n_{1}}-(\mathrm{Pb})_{n_{2}}$, are well simulated by the Morse potential [26]. Let us consider the discrete periodic structure with the periodicity cell consisting of silicon and carbon atoms.
Fig. 2 shows dependences of the effective elasticity modulus of the structures created from silicon and carbon atoms with different number and ratio of atoms in the chain. The results of discrete simulation using the Lennard-Jones potential are designated by points at all subsequent graphs in Fig. 2. The solid lines correspond to the solutions obtained using the gradient theory of elasticity (20). Numerical studies show that it is sufficient to take into account only interactions between the nearest neighboring atoms using the discrete method to study the structures. In all cases, possible relative error does not exceed $0.1 \%$. Values of potential parameters for silicon atoms: $\varepsilon_{1}=0.0025 \mathrm{eV}, \sigma_{1}=29.43 \mathrm{~A}, \quad[25]$ carbon atoms: $\varepsilon_{2}=0.0024 \mathrm{eV}, \sigma_{2}=3.37 \mathrm{~A}$ [24]. For this structure, the scale parameter of the continual model equals to $l=19.5$ A. Fig. 2, a shows dependences of the effective elasticity modulus on the scale factor, i.e. on the number of atoms of one phase in the periodicity cell. The dotted line shows the classic solution which does not allow to take into account influence of the scale effects. Fig. 2, $b$ shows comparison of the discrete and continual solutions when the number of atoms changes in the phase consisting of carbon atoms.

Si-C, $l=19.5 \AA$

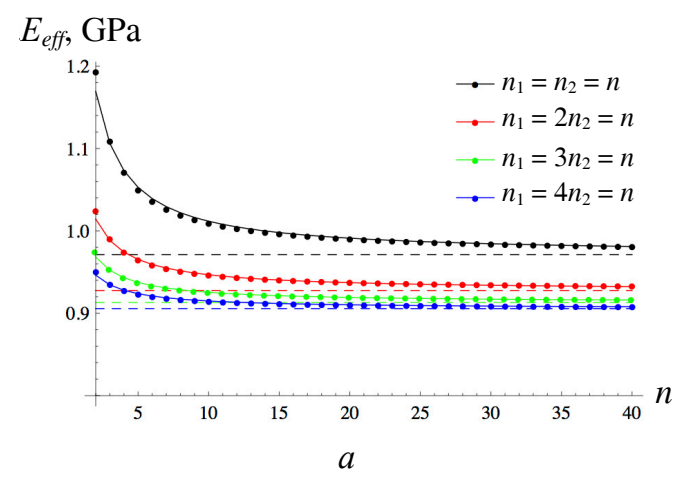

$\mathrm{Si}-\mathrm{C}, l=19.5 \AA$

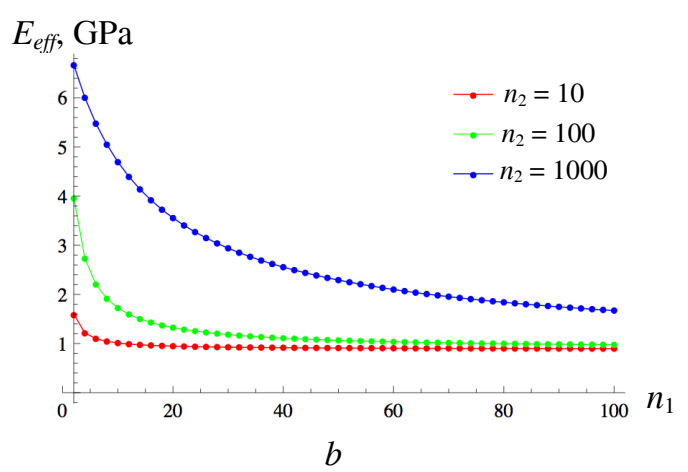

Fig. 2. Dependence of the effective elasticity modulus on the number of atoms in the Si-C structure calculated according to the discrete model using the Lennard-Jones potential (points) and the continual model (solid lines); $(a)$ - size of the cell changes, $T=\sqrt{0,5 s_{i j} s_{i j}}$, the dotted line denotes the classic solution of the elasticity theory of; $(b)$ the number of silicon atoms hanges, $n_{1}=$ const 
Fig. 3 shows dependence of the elasticity moduli of the chains created by iron and lead atoms on the number of atoms of one phase. For the simulation, we use the Morse potential with parameters for iron atoms: $\varepsilon_{1}=0.4216 \mathrm{eV}, \sigma_{1}=2.849 \mathrm{~A}, \alpha_{1}=1.3765 \mathrm{~A}^{-1}, \quad$ for lead atoms: $\varepsilon_{2}=0.2455 \mathrm{eV} \quad \sigma_{2}=2.667 \mathrm{~A}, \alpha_{2}=1.2624 \mathrm{~A}^{-1} \quad$ [26].

The scale parameter equals to $4 \mathrm{~A}$.

$$
\mathrm{Fe}-\mathrm{Pb}, l=4 \AA
$$

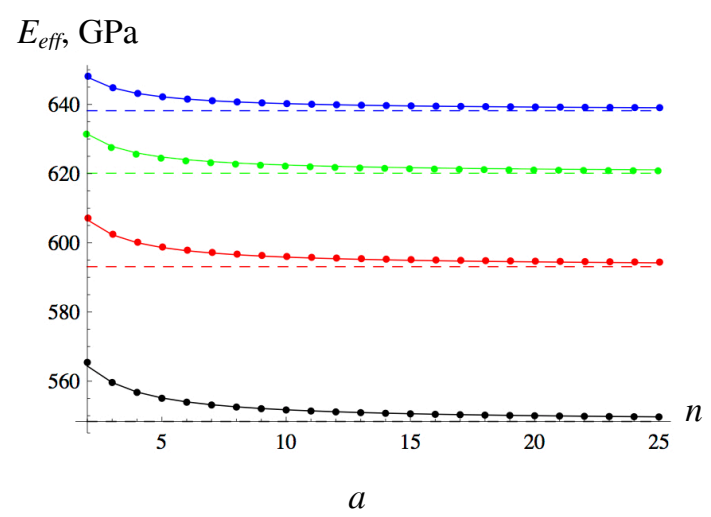

$\mathrm{Fe}-\mathrm{Pb}, l=4 \AA$

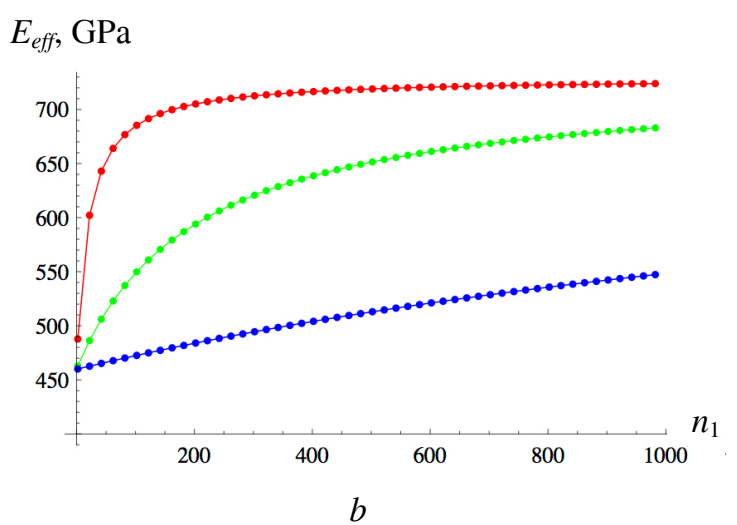

Fig. 3. Dependence of the effective elasticity modulus on the number of atoms in the $\mathrm{Fe}-\mathrm{Pb}$ structure calculated according to the discrete model using the Morse potential (points) and the continual model (solid lines); $(a)$ - size of the cell changes, $n_{1} / n_{2}=$ constant; $(b)$ the number of iron atoms changes $n_{1}$, $n_{2}=$ constant. Type and color of the lines correspond to those shown

\section{in Fig. 2, $a$ and $b$}

Table shows values of the scale parameters defined using the proposed methodology for the structures consisting of different metal atoms. We used the Morse potential for discrete simulation, parameters of the potentials were taken from [26]. The table is symmetric with respect to its central diagonal, i.e. values of the scale parameters do not change within the frameworks of the onedimensional model when the inclusion phase and matrix are interchanged.
Values of the scale parameters of the model (in angstroms) of the gradient elasticity theory identified for the chains consisting of atoms of two different metals, for example, $\mathrm{Fe}-\mathrm{Mo}, \mathrm{Fe}-\mathrm{Nb}$, etc.

\begin{tabular}{|l|c|c|c|c|c|c|c|c|c|c|c|}
\hline & $\mathrm{Mo}$ & $\mathrm{Nb}$ & $\mathrm{Ta}$ & $\mathrm{V}$ & $\mathrm{W}$ & $\mathrm{Ag}$ & $\mathrm{Au}$ & $\mathrm{Cu}$ & $\mathrm{Pb}$ & $\mathrm{Pt}$ & $\mathrm{Ni}$ \\
\hline $\mathrm{Fe}$ & 1.3 & 1.4 & 0.2 & 8 & 1.3 & 4 & 1.2 & 1.4 & 4 & 1.4 & 9 \\
\hline $\mathrm{Mo}$ & & 1.4 & 2.5 & 1.7 & 1.3 & 1.7 & 1.5 & 1.3 & 2.4 & 2 & 1.2 \\
\hline $\mathrm{Nb}$ & & & 2 & 1.7 & 1.4 & 1.7 & 1.4 & 1.4 & 2.4 & 0.9 & 1.3 \\
\hline $\mathrm{Ta}$ & & & & 1.1 & 2.2 & 1.1 & 4.8 & 0.7 & 2.2 & 2.6 & 0.3 \\
\hline $\mathrm{V}$ & & & & & 1.7 & 1.8 & 1.7 & 0.5 & 3.5 & 1.8 & 11 \\
\hline $\mathrm{W}$ & & & & & & 1.7 & 1.4 & 1.4 & 2.4 & 0.5 & 1.2 \\
\hline $\mathrm{Ag}$ & & & & & & & 1.7 & 1.3 & 4 & 1.8 & 4.5 \\
\hline $\mathrm{Au}$ & & & & & & & & 1.3 & 2.5 & 1.9 & 1.1 \\
\hline $\mathrm{Cu}$ & & & & & & & & & 6.5 & 1.5 & 1.6 \\
\hline $\mathrm{Pb}$ & & & & & & & & & & 2.5 & 4 \\
\hline $\mathrm{Pt}$ & & & & & & & & & & & 1.3 \\
\hline
\end{tabular}

Fig. 4 shows dependence of the effective elasticity modulus on the length of one phase (the number of atoms in the atomic chain). The composite includes atoms of different metals with the same number of atoms of each type $\left(n_{1}=n_{2}=n\right)$ which means that the volume content percentage of the phases equals to $50 \%$.

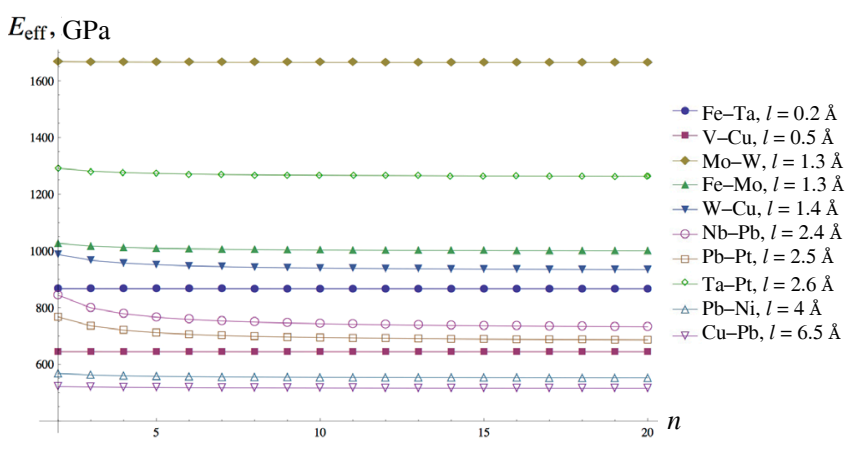

Fig. 4. Examples of dependences of the effective elasticity modulus of some two-phase composites on size of the phases (the scale effect), $n_{1} / n_{2}=1$

\section{Conclusion}

We proposed the method for determination of the scale parameters for gradient theories. The method is sufficiently accurate because direct calculations, performed using the scale parameter defined with the help of the gradient theory, give effective property values for the entire countable set of corresponding discrete composites with the relative error not exceeding $1 \%$. In the proposed method, the scale parameter actually determines the length of the interface zone in the considered two-component composites. The zone is formed in each phase in the areas adjacent to the contact boundary. Direct calculations show that, in the composites with significantly different rigidity, formation of the interface zone occurs with the help of the phase with lower rigidity, and the scale parameter of the rigid phase can be neglected. In other words, the scale parameter determines the typical scale of the material with lower rigidity. 
The results of numerical calculations show that values of the scale parameters, defined for idealized structures consisting of metal atoms, are small and do not exceed several tens of angstroms. The scale effects appear in very small cell sizes for all of the structures, i.e. for materials with high contact boundary density of the phases which is fully consistent with the physical meaning. In the systems defined by the Lennard-Jones potential, significant influence of the scale effects is predicted for the structures with the fragment of up to 40 atoms; in the systems described by the Morse potential, the scale effects make substantial impact for the periodicity fragments of up to 15 atoms. However, these effects substantially depend on the rigidity ratio of the phases. For the polymer composites with rigid nanoscale inclusions, the ratio of phase rigidity can reach 80 and the scale effects can appear in the periodicity cells that are up to tens of microns. Analysis of such structures within the frameworks of the proposed methodology is limited only to the fact that the authors have no reliable data on the potentials describing the polymer binder.

We can draw the conclusion that the forecast for the elasticity moduli is too low when we use the Lennard-Jones potential, and too high when we use the Morse potential, compared to the known typical macroscale characteristics of the materials under consideration. Apparently, the result can be refined by extending the proposed methodology to flat or three-dimensional formulation. Some refinements can be obtained using more complex and accurate interatomic interaction potentials for discrete simulation.

There are almost no scale effects in the structures where the value of the scale parameter is less than one angstrom (Fe-Ta, V-Cu). Thus, there are no scale effects in the structures where the length of local fields (using the terminology of the continual approach) is very small. On the other hand, we can observe the saturation effect for large values of the scale parameter (Fig. 4) with the volume content of $50 \%$ which does not depend on correlation between the elasticity modulus and the size of the reinforcing phase.

\section{Acknowledgements}

This work was carried out with the support of the Russian Foundation for Basic Research (Project 18-01-00553-a) and particular by the Russian Government Foundation to the Institute of Applied Mechanics of the Russian Academy of Sciences (AAAA-A17-117032010137-0).

\section{References}

1. Toupin R.A. Elastic materials with couple stresses. Arch. Rational Mech. Anal., 1962, vol. 11, pp. 385-414. DOI: 10.1007/BF00253945

2. Mindlin R.D. Micro-structure in linear elasticity. Arch. Rational Mech. Anal., 1964, vol. 16, pp. 51-78. DOI: 10.1007/BF00248490

3. Mindlin R.D. Second gradient of strain and surface-tension in linear elasticity. Int. J. Solids Struct., 1965, vol. 1, pp. 417-438. DOI: 10.1016/0020-7683(65)90006-5
4. Mindlin R.D., Eshel N.N. On first strain-gradient theories in linear elasticity. Int. J. Solids Struct., 1968, vol. 4, pp. 109-124. DOI: 10.1016/0020-7683(68)90036-X

5. Auffray N., Le Quang H., He H.C. Matrix representations for 3D strain-gradient elasticity. J. Mech. Phys. Solids, 2013, vol. 61, pp. 1202-1223. DOI: 10.1016/j.jmps.2013.01.003

6. Papanicolopulos S.A. Chirality in isotropic linear gradient elasticity. Int. J. Solids Struct., 2011, vol. 48, pp. 745-752. DOI: $10.1016 /$ j.ijsolstr.2010.11.007

7. Dell'Isola F., Sciarra G., Vidoli S. Generalized Hooke's law for isotropic second gradient materials. Proc. R. Soc. A., 2009, vol. 465, pp. 2177-2196. DOI: 10.1098/rspa.2008.0530

8. Gao X.-L., Park S.K. Variational formulation of a simplified strain gradient elasticity theory and its application to a pressurized thick-walled cylinder problem. Int. J. Solids Struct., 2007, vol. 44, pp. 7486-7499. DOI: 10.1016/j.ijsolstr.2007.04.022

9. Ma H.M., Gao X.-L., Reddy J.N. A microstructuredependent Timoshenko beam model based on a modified couple stress theory. J. Mech. Phys. Solids, 2008, vol. 56, pp. 3379-3391. DOI: 10.1016/j.jmps.2008.09.007

10. Kakunai S., Masaki J., Kuroda R., Iwata K., Nagata R. Measurement of apparent Young's modulus in the bending of cantilever beam by heterodyne holographic interferometry. Exp. Mech., 1985, vol. 25, pp. 408-412. DOI: 10.1007/BF02321341

11. Lam D.C.C., Yang F., Chong A.C.M., Wang J., Tong P. Experiments and theory in strain gradient elasticity. J. Mech. Phys. Solids, 2003, vol. 51, pp. 1477-1508. DOI: 10.1615/IntJMultCompEng.2013006064

12. McFarland A.W., Colton J.S. Role of material microstructure in plate stiffness with relevance to microcantilever sensors. J. Micromech. Microeng., 2005, vol. 15, pp. 1060-1067. DOI: 10.1088/0960-1317/15/5/024

13. Mura T. Micromechanics of defects in solids. Boston. MA: Martinus Nijhoff Publishers, 1982. 587 p.

14. Li T., Lang E. Stiffness predictions for unidirectional short-fiber composites: review and evaluation. Comp Sci Technol., 1999, vol. 59, pp. 655-71. DOI: 10.1016/S0266-3538(98)00120-1

15. Odegard G.M., Gates T.S. Constitutive modeling of nanotube/polymer composites with various nanotube orientation. Proceedings Annual Conference on Experimental and Applied Mechanics. Milwaukee, 2002, pp. 1-4.

16. Mori T., Tanaka K. Average stress in matrix and average elastic energy of materials with misfitting inclusions. Acta Metallurg., 1973, vol. 21, pp. 571-574.

17. Tibbetts G.G., McHugh J.J. Mechanical properties of vapor-grown carbon fiber composites with thermoplastic matrices. J Mater Res., 1999, vol. 14, pp. 2871-2880. DOI: 10.1007/978-94-010-0777-1_17

18. Lurie S., Volkov-Bogorodsky D., Zubov V., Tuchkova N. Advanced theoretical and numerical multiscale modeling of cohesion/ adhesion interactions in continuum mechanics and its applications for filled nanocomposites. Computational Materials Science, 2009, vol. 45, no. 3, pp. 709-714. DOI: 10.1016/j.commatsci.2008.08.010

19. Lurie S.A., Solyaev Yu.O. Simulation of the mechanical properties of nanostructured porous ceramics. Russian Metallurgy (Metally), 2013, no. 4, pp. 272-281. DOI: 10.1134/S0036029513040083

20. Lurie S., Volkov-Bogorodsky D., Leontiev A., Aifantis E. Eshelby's inclusion problem in the gradient theory of elasticity. Applications to composite materials. International Journal of Engineering Science, 2011, vol. 49, pp. 1517-1525. DOI: 10.1016/j.jjengsci.2011.05.001

21. Gusev A.A., Lurie S.A. Strain-gradient elasticity for bridging continuum and atomistic estimates of stiffness of binary 
Lennard-Jones crystals. Adv. Eng. Mater., 2010, vol. 12, pp. 529-533. DOI: 10.1002/adem.201000004

22. Altan B.S., Aifantis E.C. On the structure of the mode III crack-tip in gradient elasticity. Scripta Met., 1992, vol. 26, pp. 319-324. DOI: 10.1016/0956-716X(92)90194-J

23. Altan B.S., Aifantis E.C. On some aspects in the special theory of gradient elasticity. J. Mech. Behav. Mater., 1997, vol. 8, no. 3, pp. 231-282. DOI: 10.1515/JMBM.1997.8.3.231

24. Garcia-Perez E., Parra J.B., Ania C.O, Dubbeldam D., T. Vlugt J.H., Castillo J.M., Merkling P.J., Calero S. Unraveling the argon adsorption processes in MFI-type zeolite. J. Phys. Chem. C., 2008, vol. 112, no. 27, pp. 9976-9979. DOI: $10.1021 / \mathrm{j} 100025 \mathrm{a} 018$

25. Jeong W.K., Ho J.H., Ki O.S., Won Y.C., Ki R.B. Ordered phases of cesium in carbon nanotubes. J. Kor. Phys. Soc., 2003, vol. 43, no. 4, pp. 534-539.

26. Flahive P.G., Graham W.R. Pair potential calculations of single atom self-diffusion activation energies. Surface Science, 1980, vol. 91, pp. 449-462. DOI: 10.1016/00396028(80)90344-1

Original Russian Text:

Lurie S.A., Solyaev Y.O. Identification method of gradient models parameters of inhomogeneous structures based on discrete atomistic simulations. PNRPU Mechanics Bulletin, 2014, no. 3, pp. 89-112. DOI: 10.15593/perm.mech/2014.3.06 